\title{
Study of Some physical properties of (PVA-CuCl) composite films
}

\author{
Rezhna Mhammed Kuekha M. ${ }^{1}$, Sabah A. Salman ${ }^{2}$, Dana Mustafa Mhammed Ahmed ${ }^{1}$ \\ 1 Department of Phy sics, College of Education, University of Garmian \\ 2 Department of Physics, College of Science, University of Diy ala
}

\begin{abstract}
Composite films of (poly vinyl alcohol (PVA)) doped with $\mathrm{CuCl}$ salt were prepared using casting technique. The $\mathrm{CuCl}$ salt added to polyvinyl alcohol with different weight percentages are (0,1,1.5 and 2) wt. \%. Results showed that the absorbance increases with increase the weight percentage of $\mathrm{CuCl}$. The absorption coefficient, extinction coefficient, refractive index and real and imaginary parts of dielectric constants are increasing with increase $\mathrm{CuCl}$ concentration. The effect of annealing on the optical properties for (PVA/CuCl) composite films with different concentrations $(0,1,1.5$ and 2$)$ wt.\% of copper chloride salt were compared and the result shows that the transmittance decreased after annealing for all the composite films. The energy gap $\left(E_{g}\right)$ of all the composite films decreased after annealing. The coefficient of thermal conductivity increase by increasing the concentration of the $\mathrm{CuCl}$ salt.
\end{abstract}

\section{Introduction}

The study of composite materials, i.e., mixtures consisting of at least two phases of different chemical compositions, has been of great interest from both fundamental and practical standpoints. The macroscopic physical properties of such materials can be combined so as to produce materials with a desired average response. [1]. The properties of polymer-mineral reinforced composites are determined by the component properties (particle shape, surface area, surface chemistry, polymer microstructure) and by the preparation method and processing conditions as well. Among of preparation methods, injection molding has strong influence on the internal microstructure of polymers and in a consequence on mechanical response of the material [2]. Doping of polymers attracted the scientific and technological researchers, because of their wide applications. The dopants in polymer can change the molecular structure and hence the microstructure as well as the macroscopic properties of the polymer [3]. Polyvinyl alcohol (PVA), which is essentially made from polyvinyl acetate through hydrolysis, is easily degradable by biological organis ms and in water is a solubilized crystalline structure polymer [4]. PVA is formed by the polymerization of vinyl acetate, which is then hydrolyzed into PVA. Fig. 1 shows the chemical structure of PVA. The excellent chemical resistance and physical properties of PVA resins have led to broad industrial use [5]. PVA is a low cost hydrophilic polymer and therefore swells in the presence of water or biological fluids to form hydrogels. This property is particularly useful because it can allow for the release of drugs incorporated into these hydrogels. Since PVA has a high selectivity of water to alcohols, it exhibits low methanol permeability and has been used in some alkaline fuel cell [6-7]. PVA is an artificial polymer that has been used during the first half of the $20^{\text {th }}$ century worldwide. It has been applied in the industrial, commercial, medical, and food sectors and has been used to produce many end products, such as lacquers, resins, surgical threads, and food packaging materials that are often in contact with food [8] 


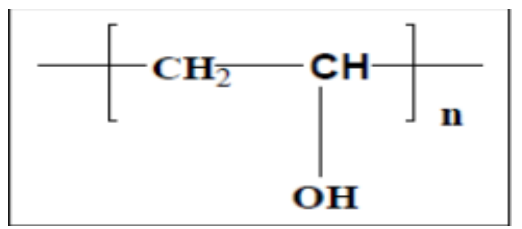

Fig.1 Chemical Structure of PVA

The purpose of this research was to discloses the investigation of the effect of doping with different concentrations of $\mathrm{CuCl}$ on the physical properties of poly(vinyl alcohol) films, compare the physical properties of polyvinyl alcohol (PVA-CuCl) composite films before and after annealing and study thermal properties of $(\mathrm{PVA} / \mathrm{CuCl})$ composite films.

\section{Materials and Method}

PVA and $\mathrm{CuCl}$ were used in this paper. The weight percentages of copper chloride are $(0,1,1.5$ and 2) wt. $\%$. The samples were prepared using casting technique. Thickness ranged between (07-13) $\mu \mathrm{m}$ measured by digital micrometer. For pure PVA film is prepared by $1 \mathrm{~g}$ of PVA and deionized water, was added into the beaker the mixture was dissolved by heating at $90^{\circ} \mathrm{C}$ for 60 minutes and with continuous stirring for 1 hour. Also for producing the polymer composites films, $\mathrm{CuCl}$ with different weight percentage was then added to produce the composite films and the solution was maintained at (80-90) ${ }^{\circ} \mathrm{C}$ under continuous stirring for 1 hour, and prepared solutions placed in Petri dish $(5 \mathrm{~cm}$ diameter $)$ to prepare the films. All the films are annealed at temperature of $\left(80^{\circ} \mathrm{C}\right)$ for $(1 \mathrm{~h})$ in air atmosphere using the electrical oven applied by Electrical laboratory furnace, LMH 07/12, S.N.: 1311360 LAC, Ltd. The optical absorption and transmission spectra of all films were recorded at room temperature before and after annealing, by UV-VIS 1800 double beam spectrophotometer provided by Shimadzu, Japanese company, in the wave length range from (190-1100)nm. The thermal properties of the samples which is the thermal conductivity were recorded for $(\mathrm{PVA} / \mathrm{CuCl})$ films by using the conventionalLee's Disk method.

\section{Result and Discussion}

\section{Thermal properties of polymers}

Thermal conductivity is defined as the property of a material's ability to conduct heat as a result of temperature difference under steady state boundary condition. It appears primarily in Fourier's Law for heat conduction [9].Thermal properties depends solely on the temperature gradient which much occur for heat to be transferred [10]. Thermal conductivity, referred to as a transport property, provides an indication of the rate at which energy is transferred by the diffusion process. It depends on the physical structure of matter, atomic and molecular, which is related to the state of the matter [11]. For the purpose of measuring thermal conductivity of $\mathrm{PVA} / \mathrm{CuCl}$ composites, the conventional Lees disk method was used Thermal conductivity $(\mathrm{k})$ may be defined as the transfer of heat from high temperature zone to low temperature zone. The law of heat transfer can be expressed as

$q=-k \frac{d T}{d s}$

Where $\mathrm{q}$ is the heat flux per unit area $\left(\mathrm{W} / \mathrm{m}^{2}\right), \mathrm{k}$ is the thermal conductivity $(\mathrm{W} / \mathrm{m} . \mathrm{K})$ and $\frac{d T}{d s}$ is the temperature gradient across medium.

Thermal conductivity $(\mathrm{k})$ is an intrinsic property of a material which relates its ability to conducting heat. The heat transfers by conduction includes transfer of energy inside a material with no any motion of the material as a whole. The used device is a modification of the standard Lee's disk method, a diagram of the device is shown in figure $(1,2)$. This consists of three copper plates $(\mathrm{A}, \mathrm{B}, \mathrm{C})$ and $(6 \mathrm{~W})$ electrical plate heater of the same diameter as the copper plates. The samples is then sited between copper plates (A) and (B) and the heater is sandwiched between two plates (B) and (C). After tightening the clamp screw to hold all the disks together, the power to the heater is switched on. The whole ass embly is placed in an enclosure to minimize the effect of draughts and fourth thermo meters are placed within the enclosure, fairly close to the device, to calculate the ambient temperature.

At the start of each determination, the power from a stabilized (DC) supply is turned on full until the average temperature of the sample (i.e. the mean of the temperature of plates (A) and (B) reached the desired value. The power is then adjusted to allow the temperature of the plates to stabilize. 
In addition to the four temperature readings, the current and voltage applied to the heater is monitored when the temperature of all parts of the device is stable. The heat $(\mathrm{H})$ supplied by the electrical heater is given $\mathrm{by}_{\mathbf{y}^{-}} \quad 1.5 \mathrm{wt} \%$ before annealing $H=V I .7$

Where $V$ is the difference potential across the heater and $I$ is the current which flows through it. Let (e) joules of energy be emitted from exposed area of the surface calculated by using equation:-

$V I \stackrel{5}{\frac{\pi}{3}} \pi r^{2} e\left(T_{A}+T_{E}\right)+2 \pi r e\left[d_{A} T_{A}+\frac{d_{s}}{2}\left(T_{A}+T_{B}\right)+d_{B} T_{B}+d_{C} T_{C}\right]$

A value the therma conductivity of the specimen (k) of thickness (d) and radius (r) is calculated using equations

$k\left[\frac{T_{B}-T_{A}}{d_{X}}\right]=e\left[T_{A}+\frac{2}{y}\left(\frac{d_{A}+\frac{d_{C}}{4}}{4}\right) T_{A}+\frac{d_{B} T_{B}}{2 F}\right]$

Where $\mathrm{ds}, \mathrm{d}_{\mathrm{A}}, \mathrm{d}_{\mathrm{B}}, \mathrm{d}_{\mathrm{C}}$ are the thickness of the sample and the disks $(\mathrm{A}),(\mathrm{B}),(\mathrm{C})$ respectively. $\mathrm{T}_{\mathrm{A}}, \mathrm{T}_{\mathrm{B}}$ and $\mathrm{T}_{\mathrm{C}}$ are the temperature of $\mathrm{N}_{\text {thele }}$ alisksh $(\mathrm{A} A)$, (B) and $(\mathrm{CC})$ above ambient. The measured values of thermal conductivity $(\mathrm{k})$ of the samples are listed in the table (1)

Table-1 Values of volu me thermal conductivity coefficient $\mathrm{k}(\mathrm{W} / \mathrm{m} . \mathrm{K})$ of $(\mathrm{PVA} / \mathrm{Cu} \mathrm{Cl})$ films with different concentrations of $\mathrm{CuCl}$ salt

\begin{tabular}{|l|l|}
\hline Samples concentration (wt. \%) & Thermal conductivity (k) (W/m. K) \\
\hline Pure (PVA) - S1 & 0.057896 \\
\hline PVA+\%1CuCl - S2 & 0.042220 \\
\hline PVA+\% $1.5 \mathrm{CuCl}-\mathrm{S} 3$ & 0.129511 \\
\hline $\mathrm{PVA}+\% 2 \mathrm{CuCl}-\mathrm{S} 4$ & 0.21272 \\
\hline
\end{tabular}

\section{Optical properties of the Polymer:}

The absorption spectra of (PVA and PVA/CuCl) films as a function of wavelength before and after annealing as shown in figure 2 . This is caused by the added $\mathrm{CuCl}$ salt which contains electrons in its outer orbits which can absorb the electromagnetic energy of the incident light and then the electrons are excited to higher energy levels, thus part of the incident light is absorbed by the substance and does not penetrate through it. On the other hand, the pure polyvinyl alcohol films are highly transparent due to the lack of free electrons (i.e. electrons are bonded to atoms), and to break these bonds photons with sufficiently high energy are needed[12]. This behavior can be explained as follows: at high wavelength the incident photons don't have enough energy to interact with atoms, the photon will transmit. When the wavelength decreases, the interaction between incident photon and material will occur and then the absorbance will increase.
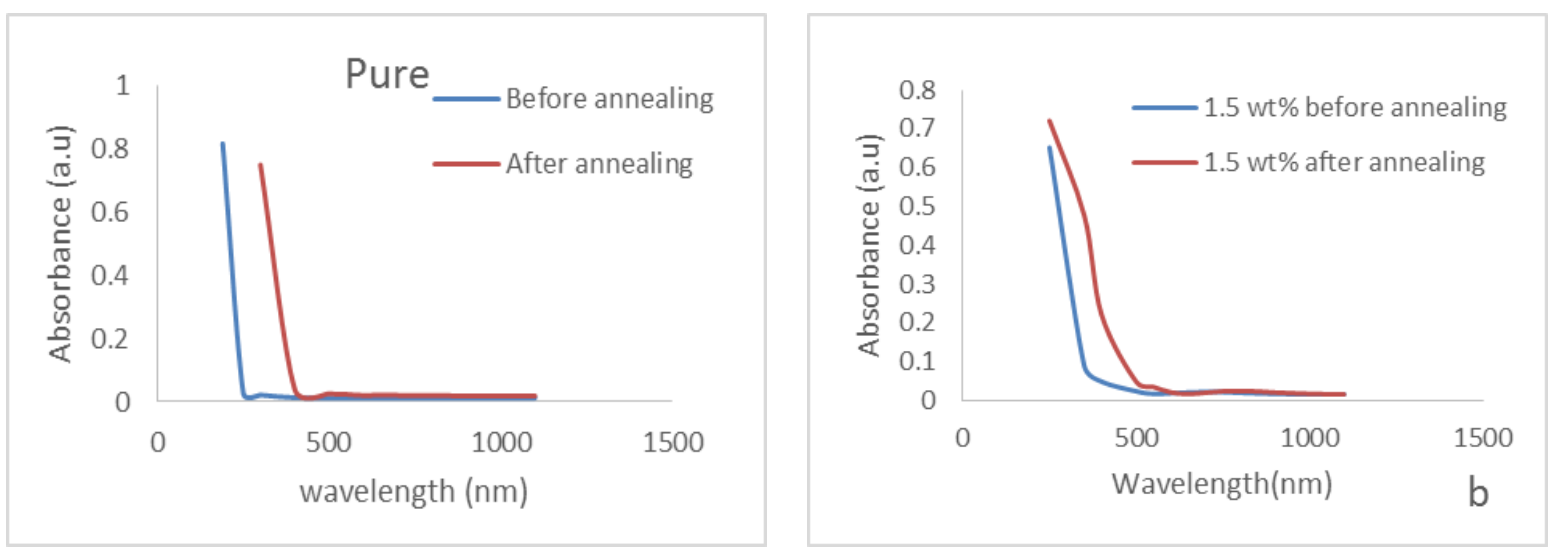

Figure (2): The absorbance of (PVA and $\mathrm{PVA} / \mathrm{CuCl}$ ) films as a function of the wavelength before annealing and after annealing with (a) 0 wt. \% and (b) 1.5 wt. \% concentrations of $\mathrm{CuCl}$ salt. 
The transmission spectrum for (PVA and PVA/CuCl) films before and after annealing as shown in fig (3). The figure shows that the transmittance increases with the increase in wavelength for all the films. It is clear that the transmittance decreased after annealing for all the films and this result is in agreement with previous reports [13-14].

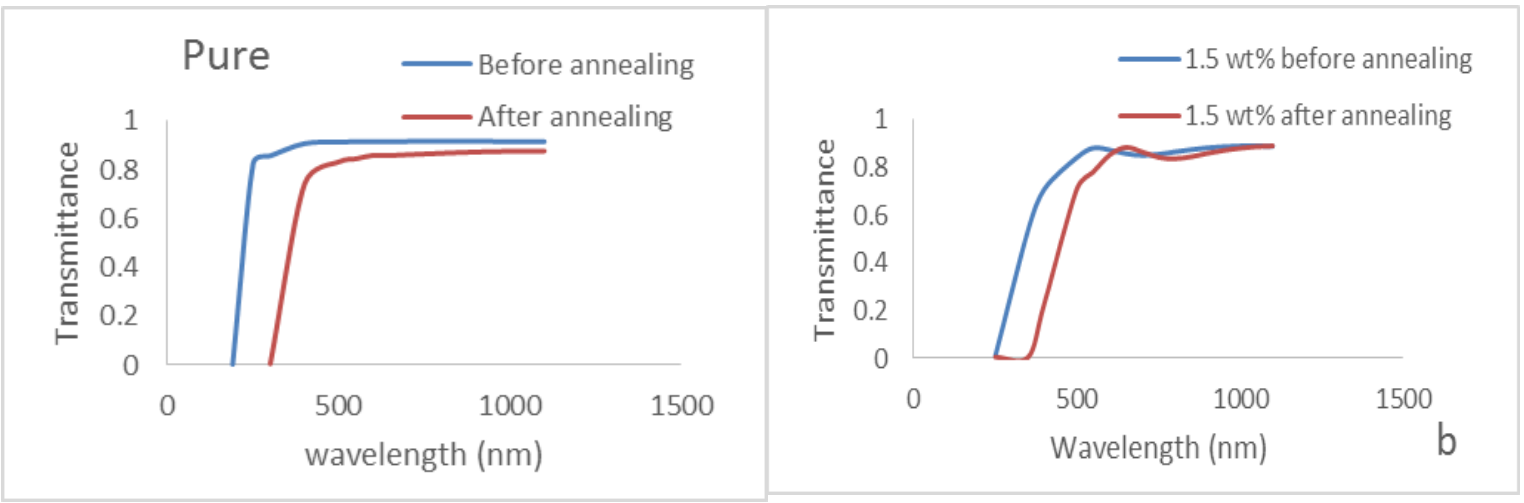

Figure (3): The transmittance of (PVA and PVA/CuCl) films as a function of the wavelength before annealing and after annealing with (a) 0 wt. \% and (b) 1.5 wt. \% concentrations of $\mathrm{CuCl}$ salt. The absorption coefficient $\alpha(\mathrm{cm})^{-1}$ is calculated by using equation (5)

$\alpha=2.303\left(\frac{A}{t}\right)$

Where $\mathrm{A}$ is the absorbance and $\mathrm{t}$ is the thickness (7-13) $\mu \mathrm{m}$ of the film. Figure 4 shows the absorption coefficient $\alpha$ for (PVA and PVA/CuCl) films as a function of the wavelength before and after annealing. One observes, through this figure, that in the shorter wavelength the absorption coeffic ient exhibit high values which means that there is a large probability of the electron transition [15] and then the absorption coefficient decreases with increase in wavelength for all the films. It is also found the absorption coefficient for $(\mathrm{PVA} / \mathrm{CuCl})$ films is less than $\left(10^{4} \mathrm{~cm}^{-1}\right)$, which explains that the electron transition is indirect. Besides, it becomes clear that the absorption coefficient increases with the increase in weight percentage of the added copper chloride salt, and that the absorption coefficient increase after annealing for all the films. However the increase of the absorption coefficient after annealing is due to its dependence on the absorbance, on the otherhand, and the decrease in defects on to the other [16].
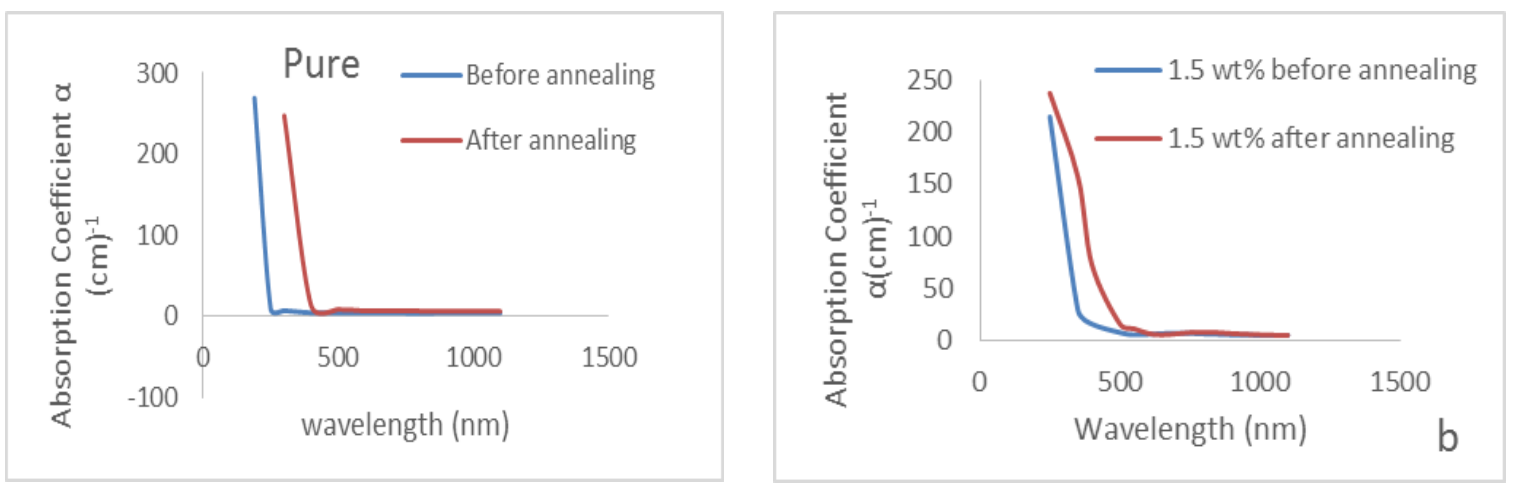

Figure (4): The absorption coefficient of (PVA and $\mathrm{PVA} / \mathrm{CuCl}$ ) films as a function of the wavelength before annealing and after annealing with (a) $0 \mathrm{wt} . \%$ and (b) $1.5 \mathrm{wt}$. \% concentrations of $\mathrm{CuCl}$ salt. The energy gap (Eg) for the allowed indirect transition can be estimated using equation:

$\alpha h v=B\left(h v-E_{g}\right)^{2}$ 
Where (B) is constant inversely proportional to amourphousity and (hv) is the photon energy. Figure-5 shows the $(\alpha h v)^{1 / 2}$ vs. hv of (PVA and PVA/CuCl) films (Tauc's Plots). For determining the energy gap (Eg) for allowed indirect transition before and after annealing, plotting a straight line which best fits the linear portion of the curve can lead to the calculation of the energy gap $\left(E_{g}\right)$ value which is the intercept of this straight line with the $\mathrm{x}$-axis where the value $(\alpha \mathrm{hv})^{1 / 2}=0$. The obtained values are shown in table (2). It is observed that the absorption edge shifts to lower energy and the energy gap (Eg) of (PVA and $\mathrm{PVA} / \mathrm{CuCl}$ ) films decreases after annealing for all the films comparing to that before annealing. The shift in the absorption edge with the heat treatment is explained by a change in the defect structure of solids. This states in the band structure is responsible for the low values of energy gap $\left(E_{g}\right)$ [17], and also because the reduction of the band gap of these films after thermal treatment and shifting the absorption properties of these organic molecules to lower energy often give rise to stability problems to oxidation [13].

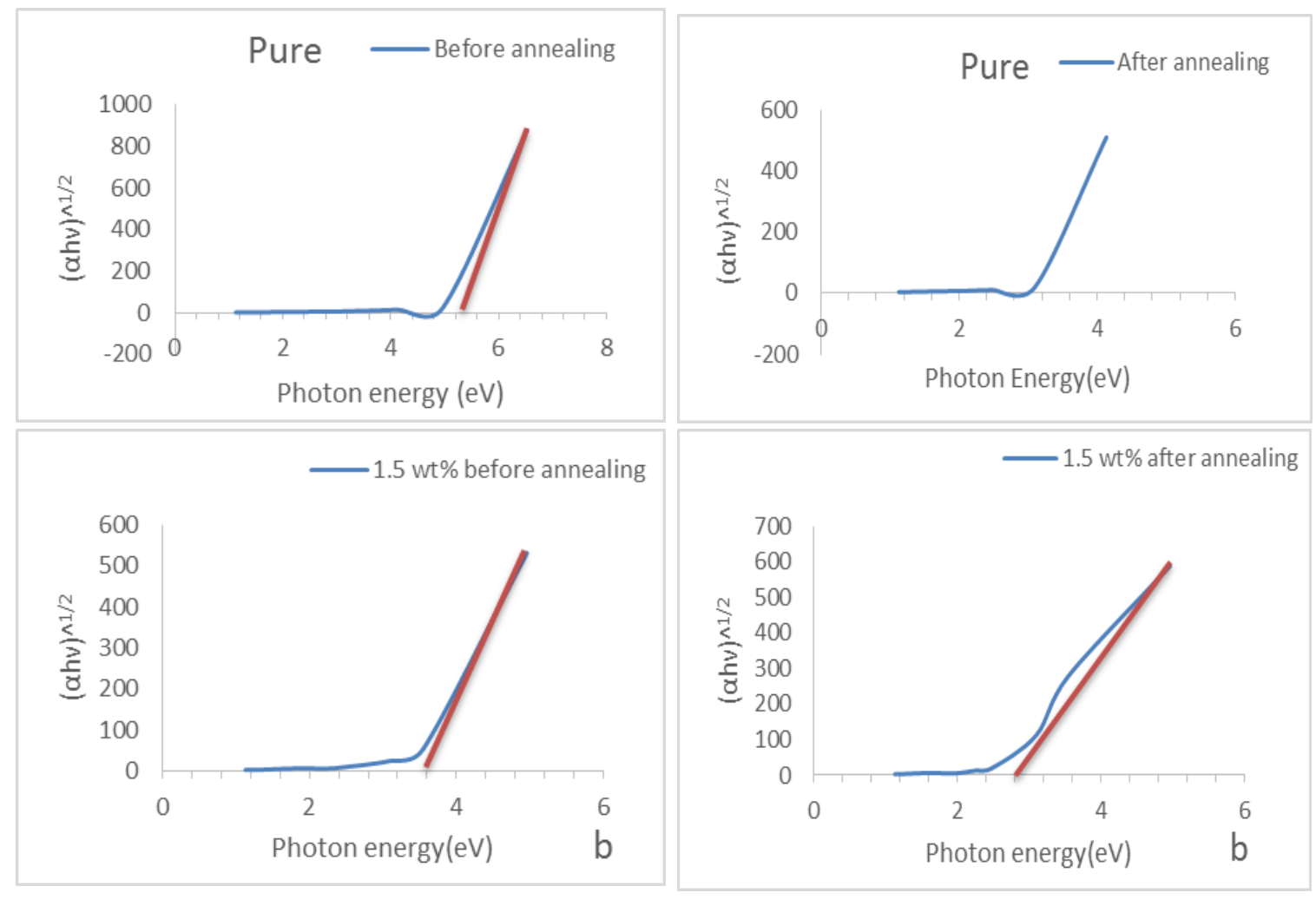

Figure (5):The energy gap $\left(E_{g}\right)$ for the allowed indirect transition of (PVA and PVA/CuCl) films as a function of the wavelength before annealing and after annealing with (a) 0 wt. \% and (b) 1.5 wt. $\%$ concentrations of $\mathrm{CuCl}$ salt.

Table (2) Values of energy gap $\left(E_{g}\right)$ for the allowed indirect transition of (PVA and PVA/CuCl) films with $0 \mathrm{wt} . \%$ and $1.5 \mathrm{wt} . \%$ concentrations of $\mathrm{CuCl}$ salt before and after annealing.

\begin{tabular}{|l|l|l|}
\hline Concentration (wt. \%) & Before annealing $E_{g}(\mathrm{eV})$ & After annealing $E_{g}(\mathrm{eV})$ \\
\hline 0 & 5.2 & 3.25 \\
\hline 1.5 & 3.4 & 2.9 \\
\hline
\end{tabular}

Figure- 6 shows the change of refractive index of (PVA and PVA/CuCl) films as a function of wavelength. The refractive index (n) is calculated from equation [18]:

$n=\sqrt{\frac{4 R-k^{2}}{(R-1)^{2}}}-\frac{(R+1)}{(R-1)}$ 
Where $\mathrm{R}$ is the reflectance and $\mathrm{k}$ is the extinction coefficient. It is clear that the refractive index increased after annealing for all the films.
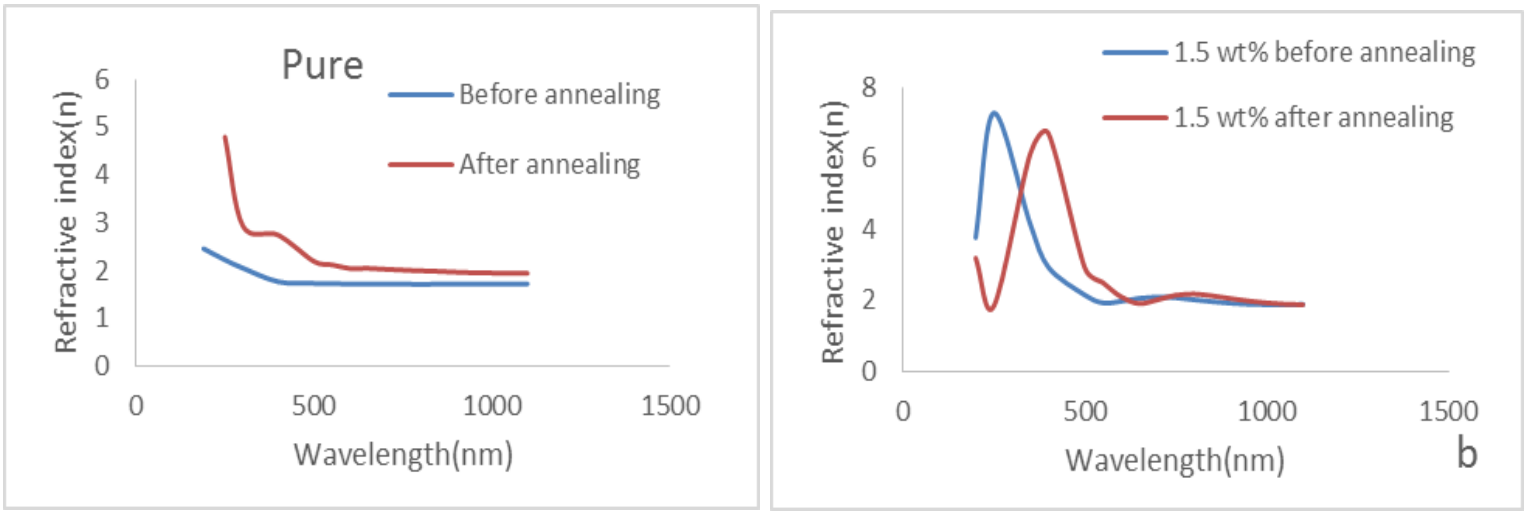

Figure (6):The refractive index of (PVA and PVA/CuCl) films as a function of the wavelength before annealing and after annealing with (a) 0 wt. \% and (b) 1.5 wt. \% concentrations of $\mathrm{CuCl}$ salt.

Figure- 7 shows the change of extinction coefficient of (PVA and PVA/CuCl) films as a function of the wavelength before and after annealing. The extinction coefficient $(k)$ is calculated from equation [19] $k=\frac{\alpha \lambda}{4 \pi}$

Where $(\lambda)$ is the wavelength of incident ray.

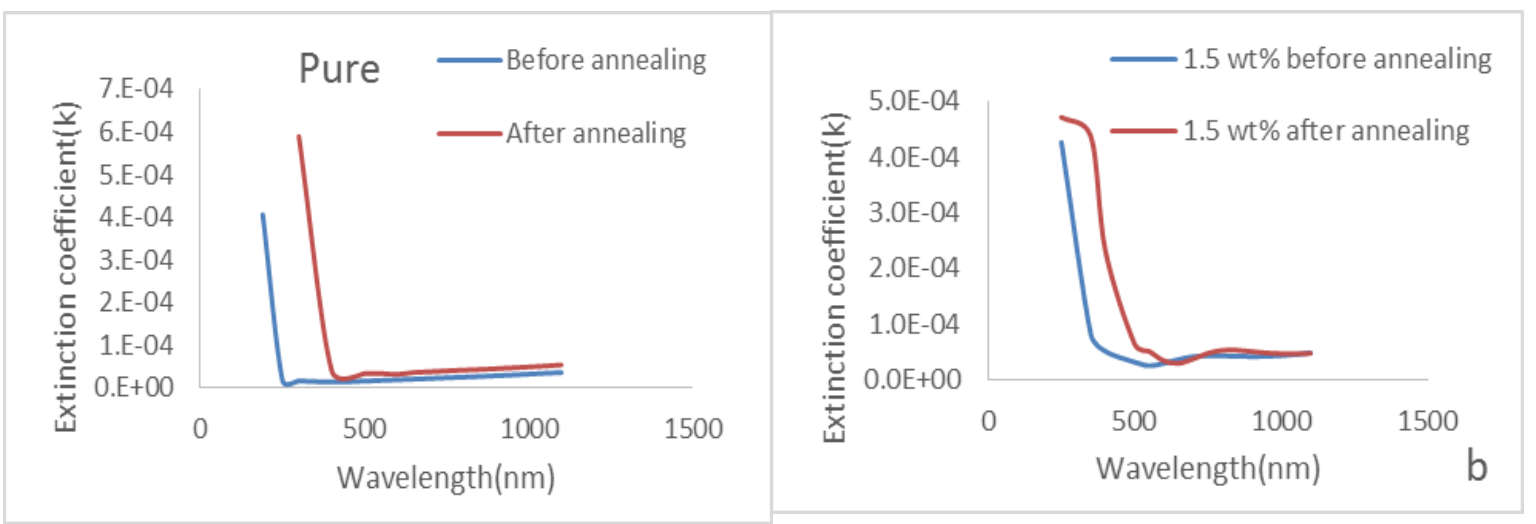

Figure (7):The extinction coefficient of (PVA and $\mathrm{PVA} / \mathrm{CuCl}$ ) films as a function of the wavelength before annealing and after annealing with (a) $0 \mathrm{wt}$. \% and (b) $1.5 \mathrm{wt}$. \% concentrations of $\mathrm{CuCl}$ salt.

From the figure 7 its clear that the extinction coefficient increased after annealing for all the films. This is attributed to high absorption coefficient because the behavior of extinction coefficient is nearly similar to the corres ponding absorption coefficient.

Figures (8) and (9) show the change of the real and imaginary parts of dielectric constant of (PVA and PVA/CuCl) films as a function of the wavelength before and after annealing. The real $\left(\varepsilon_{1}\right)$ and imaginary $\left(\varepsilon_{2}\right)$ parts of dielectric constant can be expressed by the following equation [19]: $\varepsilon_{1}=n^{2}-k^{2}$

$\varepsilon_{2}=2 n k$ 
Figure (8):The real part of dielectric constant of (PVA and PVA/CuCl) films as a function of the wavelength before annealing and after annealing with (a) 0 wt. \% and (b) 1.5 wt. \% concentrations of $\mathrm{CuCl}$ salt.

It can be seen that the real and imaginary parts of dielectric constant increased after annealing for all the films. This behavior is similar to (n) and (k) because $\left(\varepsilon_{1}\right)$ depends on $\left(n_{2}\right)$ due to low value of $\left(k_{2}\right)$, while $\left(\varepsilon_{2}\right)$ is dependent on $(\mathrm{k})$ value which depends on the $(\alpha)$. The real and imaginary parts of dielectric constant indicate the same pattern and the values of real part are higher than the values of imaginary part [13, 14].

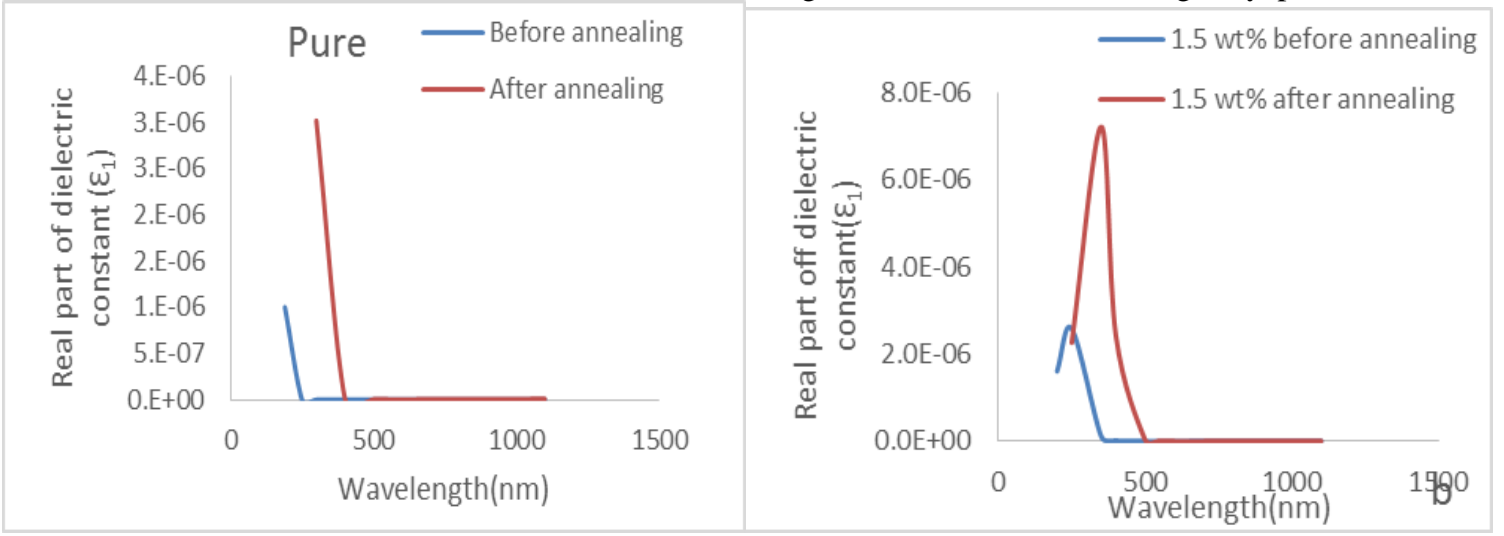

Figure (9):The imag inary part of dielectric constant of (PVA and PVA/CuCl) films as a function of the wavelength before annealing and after annealing with (a) 0 wt. \% and (b) 1.5 wt. \% concentrations of $\mathrm{CuCl}$ salt.

\section{Conclusion}

In this work, the effect of $\mathrm{CuCl}$ impurity and annealing on the optical and thermal properties of PVA films has been studied, using solution cast technique. The absorption coefficient, extinction coefficient, refractive index and real and imaginary parts of dielectric constants are increasing with increase $\mathrm{CuCl}$ concentration. The effect of annealing on the optical properties for $(\mathrm{PVA} / \mathrm{CuCl})$ composite films with different concentrations $(0,1,1.5$ and 2) wt.\% of copper chloride salt were compared and the result shows that the transmittance decreased after annealing for all the composite films. The energy gap $\left(E_{g}\right)$ of all the composite films decreased after annealing. The coefficient of thermal conductivity increase by increasing the concentration of the $\mathrm{CuCl}$ salt.

\section{References}

[1] Canan Aksu Canbay and Ayşe Aydogdu, "Microstructure, Electrical and Optical Characterization of $\mathrm{ZnO}-\mathrm{NiO}-\mathrm{SiO}_{2}$ Nanocomposite Synthesized by Sol- Gel Technique", Turkish Journal of Science \& Technology, Vol. 4, No 2, pp. 121- 126. (2009).

[2] K. Bula, T. Jesionowski and S. Borysiak, "Effect of injection molding conditions on composite properties based on PBT with $\mathrm{SiO}_{2}$ and MMt Nanofillers", Proceedings of the Conference of Multiphase Polymers and Polymer Composites: From Nanoscale to Macro Composites", Paris-Est, Creteil Uniersity, Vol. 63, pp.98-105, France, (2011).

[3] A. Tawansi, M. D. Migahed and M. I. A. El-Hamid, "Part B: Poly mer physics", Journal of Polymer Science, Vol. 24, No. 12, p. 2631, (1986).

[4] Razzak, M.T.; Darwis, D.; Zainuddin; Sukirno. "Irradiation of polyvinyl alcohol and polyvinyl pyrrolidone blended hydrogel for wound dressing." Radiat. Phys. Chem., 62, 107-113. (2001)

[5] J. I. Kroschwitz, "Encyclopedia of Polymer Science and Engineering". New York: John Wiley and Sons Inc. (1989).

[6] C.C.Yang, S.J. Chiu and W.C. Chien, J. "All solid-state electric double-layer capacitors based on alkaline polyvinyl alcohol polymer electrolytes” Journal of Power Sources. Vol. 152 pp. 303-310 (2006). 
[7] C.C. YangJ. Membr. Sci., "Synthesis and characterization of the cross-linked $\mathrm{PVA}_{\mathrm{T}} \mathrm{TiO}_{2}$ composite polyme membrane for alkaline DMFC". Journal of Membrane Science Volume 288, Issues 1-2, pp. 51-60. (2008).

[8] Demerlis, C.C.; Schoneker, D.R. "Review of the oral toxicity of polyvinyl alcohol (PVA)". Food Chem. Toxicol., 41, 319-326. (2003).

[9] A.L.Saroj, R.K.Singh. "Thermal, dielectric and conductivity studies on PVA/Ionic liquid [EMIM][EtSO4] based polymer electrolytes". Journal of Physics and Chemistry of Solids

Vol. 73, Issue 2, , pp.162-168 (2012).

[10] J.Malathia, M.Kumaravadivel, G.M.Brahmanandhan, M.Hema, R.Baskaran, S.Selvasekarapandian. "Structural, thermal and electrical properties of PVA-LiCF3SO3 poly mer electrolyte". Journal of NonCrystalline Solids Vol. 356, Issue 43, pp. 2277-2281 (2010).

[11] Theodore L. Bergman, Adrienne S. Lavine, Frank P. Incropera and David P: Fundamen tals of Heat and Mass Transfer by. DeWitt Published by AiPi (2009) .

[12] El-Dahshan M. E., Introduction to Material Science and Engineering, 2nd Ed., King Saud University Press, Saudi Arabia, (2002).

[13] O. G. Abdullah and D. R. Saber, "Optical absorption of Polyvinyl alcohol films doped with Nicke1 Chloride", Applied Mechanics and materials, Vol. 110-116, pp. 177-182, (2012).

[14] M. Abdallh, O. Hamood and E. Yousif, "Study the optical properties of Poly (vinyl alcohol) doped Copper Chloride", Journal of Al-Nahrain University, Vol. 16, No. 1, pp. 17-20, (2013).

[15] J. I. Langford and A. J. C. W ilson, "Scherrer after sixty years: a survey and some new results in the determination of crystallite size", Journal of Applied Crystallography, Vol. 11, No. 2, pp. 102-113, (1978).

[16] D. K. Dwivedi, V. Kumar, M. Dubey and H. P. Pathak, " Structural, electrical and optical investigations of CdSe nanoparticles", Chalcogenide Letters, Vol. 8, No. 9, pp. 521 - 527, (2011).

[17] N. Fadhil Habubi, Z. AI-Ramadhan and R. Ismaee khaleel "Annealing effect on optical Properties of Poly (Vinyl Chloride) modified by Nickel eyhyl xanthate chelate complex" Proceeding of 3rd Scientific conference of the College of Science, University of Baghdad, pp. 2080-2087, March (2009).

[18] K. L. Chopra and Kaur I., Thin film Device Applications, Plenum Press, New York, (1983).

[19] Klingshirn C., Semiconductor Optics, Springer-Verlag Berlin Heidelberg, (1997) 